\title{
Expression of a tumor-associated gene, LASS2, in the human bladder carcinoma cell lines BIU-87, T24, EJ and EJ-M3
}

\author{
QINGHUA ZHAO ${ }^{1 *}$, HAIFENG WANG ${ }^{2 *}$, MINGYING YANG $^{2}$, \\ DELIN YANG $^{2}$, YIGANG ZUO ${ }^{2}$ and JIANSONG WANG ${ }^{2}$ \\ Departments of ${ }^{1}$ Gynaecology and ${ }^{2}$ Urology, The Second Affiliated Hospital of Kunming Medical University, \\ Yunnan Institute of Urology, Kunming 650101, P.R. China
}

Received November 20, 2012; Accepted January 2, 2013

DOI: $10.3892 /$ etm.2013.892

\begin{abstract}
Homo sapiens longevity assurance homolog 2 of yeast LAG1 (LASS2), a metastasis suppressor gene of human cancer, is the most abundantly expressed member of the ceramide synthase gene family. Expression of LASS2 has been reported in carcinomas of the prostate, liver and breast. However, there has been no report on the expression of LASS2 in human bladder cancer cell lines. In order to investigate the expression and potential role of this new tumor metastasis supressor gene in human bladder cancer, we compared the proliferation, metastasis and invasion among the BIU-87, T24, EJ and EJ-M3 human bladder cancer cell lines. The mRNA expression levels of the LASS2 gene were examined using real-time quantitative PCR (qPCR). The expression levels of LASS1 and LASS3 mRNA were used as references. The protein expression level of the LASS2 gene was detected using western blotting. The most aggressive of these four human cancer cell lines was observed to be EJ-M3. The expression of LASS2 mRNA was significantly correlated with diverse proliferation, metastasis and invasion. The expression levels of LASS1 and LASS3 mRNA were not correlated with these parameters. At the protein level, we observed that the more aggressive the cancer cell line, the lower the LASS2 protein expression level. Therefore, LASS2 expression may be correlated with the development and progression of human bladder cancer and may be a prognostic indicator for this cancer.
\end{abstract}

\section{Introduction}

Bladder cancer is one category of common genitourinary cancers and recurrence with metastasis is the main reason for

Correspondence to: Professor Jiansong Wang, Department of Urology, The Second Affiliated Hospital of Kunming Medical University, 374 Dianmian Avenue, Kunming, Yunnan 650101, P.R. China

E-mail: jiansongwang@126.com

*Contributed equally

Key words: bladder cancer, Homo sapiens longevity assurance homolog 2 of yeast LAG1, expression, biological characteristics treatment failure (1). Metastases are the end result of tumor progression and the most common cause of mortality in cancer patients. The process of metastasis is multistep and interruption of this process at any individual step may arrest the metastatic cascade (2). Therefore, it is important to investigate the biological mechanisms contributing to the development and metastatic movement of bladder cancer.

Tumor metastasis suppressor genes are a relatively new class of genes. These genes, which include NM23 (3-7), KISS-1 (8-11) and RhoGDI2 (12-14), reduce the metastatic ability of cancer cells at orthotopic sites,. However, the mechanism of action and role in human cancer remains unknown for the majority of these genes.

The LASS (longevity assurance homolog) family members are highly conserved from yeasts to mammals. Homo sapiens longevity assurance homolog 2 of yeast LAG1 (LASS2), also known as tumor metastasis suppressor gene 1 (TMSG1, GenBank accession number AF189062), is a gene isolated from a human liver cDNA library by the laboratory of Shanghai Medical College, Fudan University (Shanghai, China), and it is a human homolog of the yeast (Saccharomyces cerevisiae) longevity assurance gene, LAG1. LASS2 has been observed to correlate with the degree of invasion and recurrence in carcinomas of the prostate $(15,16)$, liver (17) and breast (18). However, there has been no report on the expression of LASS2 in human bladder cancer cell lines.

In our previous study, we demonstrated that LASS2-negative bladder cancer was associated with poor clinical prognosis. The expression of LASS2 mRNA was significantly correlated with clinical stage $(\mathrm{P}<0.001)$, depth of tumor invasion $(\mathrm{P}<0.001)$ and recurrence $(\mathrm{P}<0.001)(19)$.

In order to fully understand the biological importance of LASS2, we examined the mRNA and protein expression of LASS2 in human bladder cancer cell lines (BIU-87, T24, EJ and EJ-M3) with diverse proliferation and invasion potential, and analyzed the potential role of the tumor metastasis supressor gene LASS2 in these human bladder cancer cell lines.

\section{Materials and methods}

Cell lines and cell culture. The EJ, T24 and BIU-87 human bladder cancer cell lines were preserved by our department 
(Department of Urology, the Second Affiliated Hospital of Kunming Medical University, Yunnan Institute of Urology, China). The highly invasive human bladder carcinoma EJ-M3 cell line was established in previous studies $(20,21)$. The BIU-87, T24, EJ and EJ-M3 cell lines were cultured in DMEM supplemented with $10 \%$ fetal bovine serum and incubated in $5 \% \mathrm{CO}_{2} / 95 \%$ air at $37^{\circ} \mathrm{C}$.

Cell proliferation assay. The growth of the BIU-87, T24, EJ and EJ-m3 cells was evaluated by a cell proliferation assay. Briefly, cells $\left(1 \times 10^{4}\right)$ were plated in seven 24-well culture plates after the cell count. The cells were harvested on days 1 , 2, 3, 4, 5, 6 and 7 for cell counting experiments, and the values were normalized to untreated controls. This experiment was repeated three times. We then created growth curves and compared the proliferation ability among the four cell lines.

Matrigel invasion assay. The assay was carried out according to the method of Girnita et al (22). The invasive ability of BIU-87, T24, EJ and EJ-M3 cells was evaluated using a Matrigel invasion assay. BD BioCoat Matrigel Invasion Chambers with $8-\mathrm{mm}$ pore size PET membranes (BD Biosciences, San Jose, CA, USA) for 24-well plates were prepared by hydrating for $2 \mathrm{~h}$ at $37^{\circ} \mathrm{C}$. A total of $2 \times 10^{5}$ cells in $0.2 \mathrm{ml}$ were seeded into each insert. After culturing for $12 \mathrm{~h}$, the invasion chamber was removed and the medium in the top wells was aspirated and cells on the upper surface of the membranes were removed with cotton swabs. The invading cells which remained attached to the lower side of the membrane were removed by flushing with a pipette before migrating cells present in the bottom chambers were labeled. The fluorescence intensities were plotted on a standard histogram and the number of invading cells was calculated. All experiments were performed in triplicate. Data were expressed as the number of invaded cells.

Total RNA isolation and real-time quantitative PCR $(q P C R)$. According to the manufacturer's instructions, total RNA was extracted using TRIzol (Invitrogen, Carlsbad, CA, USA). First-strand cDNA was synthesized in a volume of $20 \mu \mathrm{l}$ using $1 \mu \mathrm{g}$ total RNA and TaqMan reverse transcription reagents (Applied Biosystems, Foster City, CA, USA). The target gene sequences were obtained from the National Center for Biotechnology Information GenBank databases. The purity of RNA samples was determined by the $\mathrm{OD}_{260} / \mathrm{OD}_{280}$ (between 1.7 and 2.0) and the $\mathrm{OD}_{260} / \mathrm{OD}_{230}$ (>1.7) values and by analysis of the ribosomal RNA band integrity by conventional denaturing agarose RNA electrophoresis (23). PCR was performed with an ABI PRISM 7000 (Applied Biosystems) and 2X qPCR MasterMix (Eurogentec, Seraing, Belgium) according to the manufacturer's instructions. To quantify target mRNA levels, LASS1, LASS2 and LASS3 Genes Expression assays were purchased from Applied Biosystems. The primer sequences were as follows: LASS1 forward 5'-CACACACATCTTTCGGCCC-3', and reverse 5'-ACCTGGCAGCATCTCTAGGC-3'; LASS2 forward 5'-TCTCCTGGTTTGCCAATTACG-3', reverse 5'-CCGGGCAGGGACCCTCATCA-3'; LASS3 forward 5'-GAGCGCCAGGTTGAAAGATG-3', and reverse 5'-GGAATTTCTGCAGCCTGCA-3'. All primers spanned

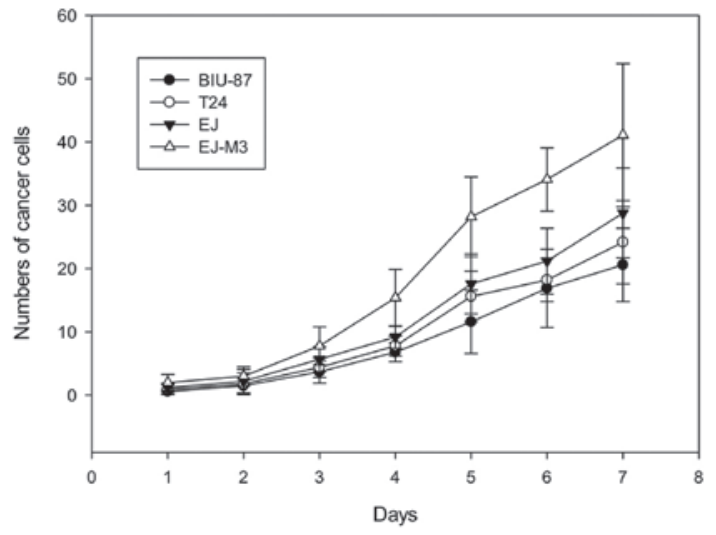

Figure 1. Growth curves of BIU-87, T24, EJ and EJ-M3 cells. The EJ-M3 cell proliferation was the fastest $(\mathrm{P}<0.001$, vs. BIU-87, T24 and EJ) and BIU-87 cell proliferation was the slowest $(\mathrm{P}<0.05$ vs. T24, EJ and EJ-M3).

an intron to ensure discrimination between cDNA and genomic DNA. The relative amount of specific mRNA was normalized to GAPDH using the following primer sequences: forward 5'-GGTCTCCTCTGACTTCAACA-3', and reverse 5'-GAGGGTCTCTCTCTTCCT-3'. All PCR reactions were run in duplicate and were performed with 40 cycles. A dilution series was carried out for each gene and the $18 \mathrm{~S}$ ribosomal subunit was used as an internal control. qPCR analysis was carried out using the $2^{-\Delta \Delta \mathrm{Ct}}$ method (24).

Western blot analysis. Western blots of whole-cell lysates from a known number of cells were prepared. The whole-cell lysates were made by lysing cells in the buffer. Protein (50 mg) was separated by SDS-PAGE gels and transferred to a membrane. LASS2 was detected in $100 \mu \mathrm{g}$ of protein from whole cell extracts, according to the procedure described by Baron et al (25) and Calogero et al (26), and actin was used as a loading control. The primary and secondary antibodies were purchased from Santa Cruz Biotechnology (Santa Cruz, CA, USA). The protein was visualized by enhanced chemoluminescence (ECL; Santa Cruz) and normalized with respect to the actin content of each sample.

Statistical analysis. The results were expressed as mean \pm the standard error of the mean (SEM) of at least three separate experiments. Statistical significance was assessed using a two-tailed unpaired Student's t-test. The correlation between gene expression and potential causative variables, were evaluated with the Chi-square test. $\mathrm{P}<0.05$ was considered to indicate a statistically significant result. Each statistical analysis was performed using the SPSS 11.0 software for Windows (SPSS Inc., Chicago, IL, USA).

\section{Results}

Growth curves. The population-doubling time of BIU-87, EJ, T24 and EJ-M3 cell lines were $31.7 \pm 0.1,27.6 \pm 0.2,28.5 \mathrm{~h} \pm 0.1$ and $20.8 \pm 0.2 \mathrm{~h}$, respectively. The cell growth rate was significantly different between EJ-M3 and the other three cell lines $(\mathrm{P}<0.05)$. There was no significant difference between the growth rates of BIU-87 and T24 ( $>00.05)$. The growth curves are shown in Fig. 1. 


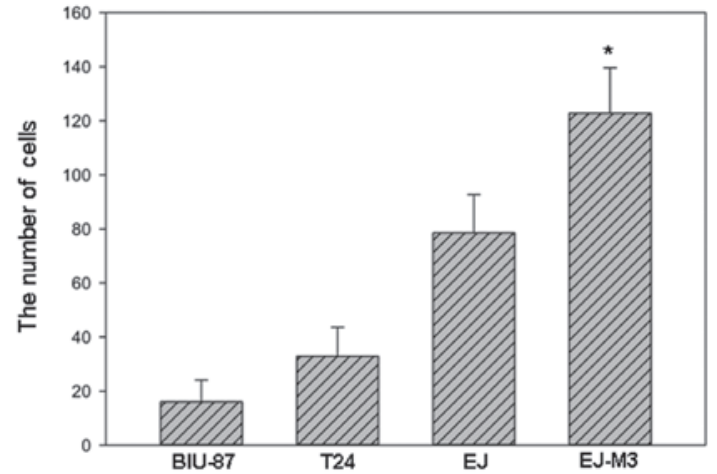

Figure 2. Numbers of EJ-M3, EJ, T24 and BIU-87 cells that attached to the lower side of the membrane. ("P<0.05, vs BIU-87, T24 and EJ).

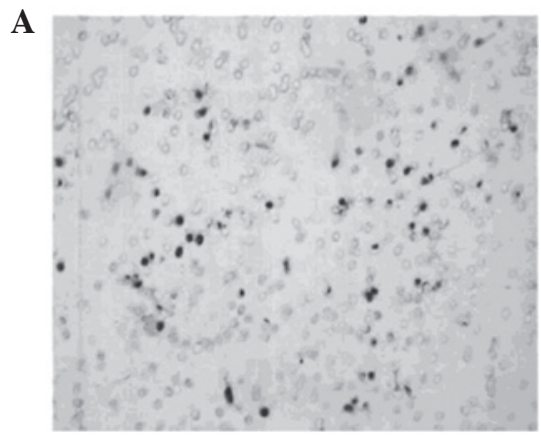

B

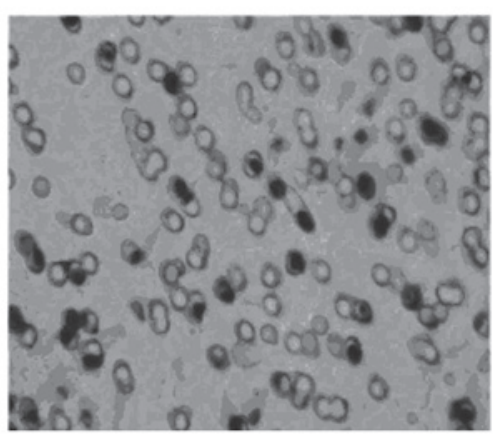

Figure 3. Invasion assay. (A) EJ cells invasion, x100 magnification; (B) EJ cells invasion, x200 magnification.

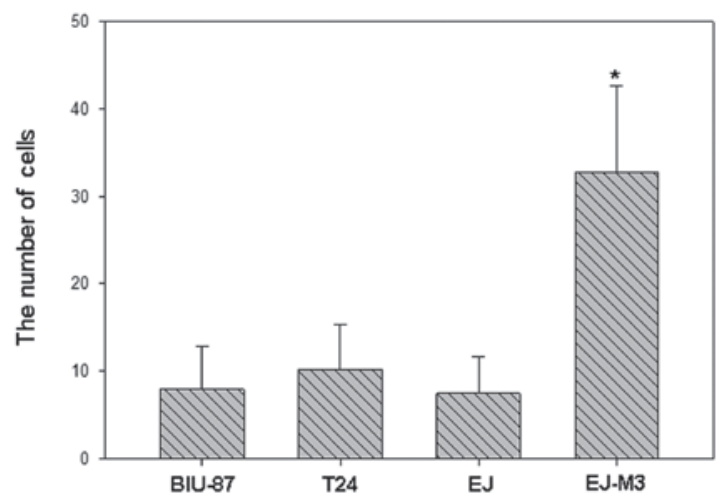

Figure 4. Numbers of EJ-M3, EJ, T24 and BIU-87 cells that invaded into the 24-well plates ( $\mathrm{P}<0.001$, vs. BIU-87, T24 and EJ).

In vitro invasion. The numbers of EJ-M3, EJ, T24 and BIU-87 cells that attached to the lower side of the membrane

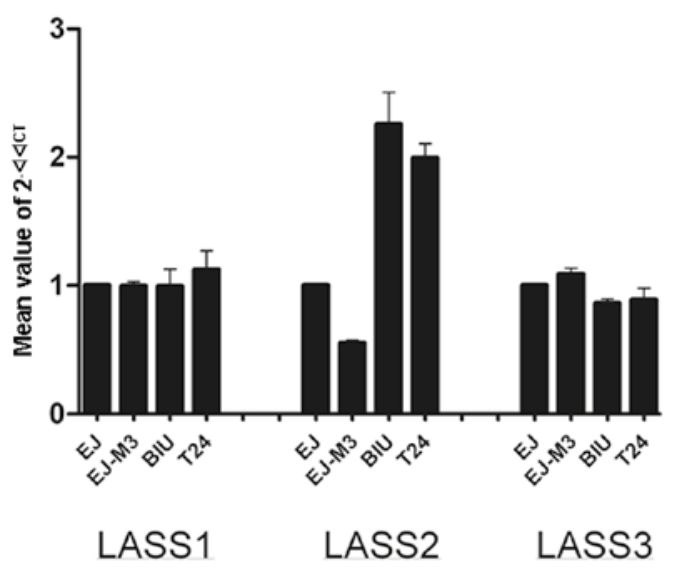

Figure 5. LASS1, LASS2 and LASS3 mRNA expression analysis. LASS1, LASS2 and LASS3 mRNA expression of BIU-87, EJ-M3, T24 and EJ cells was detected by quantitative real-time PCR.

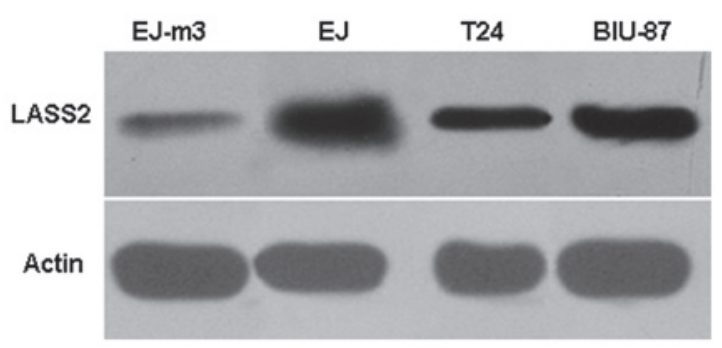

Figure 6. LASS2 protein expression analysis. LASS2 protein expression of BIU-87, EJ-M3, T24 and EJ cells was detected by western blotting. The difference in LASS2 protein expression among the four cell lines was statistically significant.

were $122.8 \pm 16.8,78.6 \pm 14.1,32.9 \pm 10.7$ and $16.0 \pm 8.1$, respectively. The numbers of EJ-M3, EJ, T24 and BIU-87 cells that invaded into the 24 -well plate were $32.8 \pm 9.8,7.5 \pm 4.2$, $10.2 \pm 5.1$ and $8.0 \pm 4.9$, respectively. Using the method of analysis of variance (ANOVA), we demonstrated that there was no statistic difference of invasiveness between EJ, T24 and BIU-87. However, the invasiveness of EJ-M3 was significantly different from that of EJ, T24 and BIU-87 $(\mathrm{P}<0.001)$ and are shown in Fig.s 2-4.

qPCR analysis of LASS1, LASS2 and LASS3 mRNA expression in the EJ-M3,EJ, T24 and BIU-87 cell lines. Prior to the quantitative analysis, optimization procedures were carried out for qPCR reactions on EJ-M3, EJ, T24 and BIU-87 cell lines, using specific primers for human LASS2, LASS2 and LASS3 genes, separately. Since LASS1, LASS2 and LASS3 have been reported to be expressed at the mRNA level in normal human tissues, we used LASS2 expression as a control in qPCR reactions. To optimize our quantitative technique, a cDNA synthesized from the cell sample was used in serial dilution and a PCR efficiency close to $100 \%$ was obtained. After optimization of the qPCR, expression of LASS2, LASS2 and LASS3 genes were analyzed using qPCR in the bladder carcinoma cell lines EJ-M3, EJ, T24 and BIU-87. The result is shown in Fig. 5. 
The differences among the four bladder carcinoma cell lines were calculated using the ANOVA method. The differences in LASS2 mRNA expression levels among the four cell lines were statistically significant $(\mathrm{P}<0.001)$. LASS1 and LASS3 expression levels among the four cell lines were not statistically significant $(\mathrm{P}>0.05)$.

Western blot analysis of LASS2 protein expression in the EJ-M3, EJ, T24 and BIU-87 cell lines. The expression of LASS2 protein was observed in the four bladder carcinoma cell lines, EJ-M3, EJ, T24 and BIU-87. The highest expression level of LASS2 was detected in the human bladder carcinoma cell line EJ-M3 by western blot analysis. Moderate LASS2 protein expression was observed in EJ and T24 cell lines, and weak LASS2 expression was observed in the BIU-87 cell line by western blotting. The result is shown in Fig. 6 .

\section{Discussion}

The present study identified the presence of LASS2 mRNA and protein in four bladder carcinoma cell lines. The expression levels of LASS2 among these human bladder cancer cell lines, which have diverse reproductive activity and invasive abilities, are distinct at the mRNA and protein level.

It is well known that an imbalance of cellular growth regulation results from certain genetic changes in the oncogenic process, which lead to uncontrolled tumor growth. However, unrestrained proliferation does not, by itself, result in invasion and metastasis. Certain additional genetic changes are required for tumor cells to be able to invade and metastasize (27). A tumor metastasis suppressor gene that is involved in tumor cell metastasis has been confirmed (28).

LASS2 has previously been demonstrated to function as a tumor metastasis suppressor gene. Previous studies have shown that LASS2 gene plays a key role in carcinomas of the prostate $(15,16)$, liver (17) and breast (18).Chen et al (17) observed that transfection of LASS2 by lipofectamine inhibited the invasion and metastasis of a highly metastatic liver cancer cell line, HCCLM3. Su et al $(15,16)$ demonstrated that overexpression of TMSG-1 (LASS2) inhibits the proliferation, anchorage-independent growth and invasion of a highly metastatic prostate cell line, PE-3M-1E8. We observed that LASS2-negative bladder cancer was associated with poor clinical prognosis and the expression of LASS2 was significantly correlated with clinical stage, depth of tumor invasion and recurrence in our preliminary studies (19).

This is the first study investigating the expression of LASS2 in human bladder carcinoma cell lines. In this study, we examined the protein and mRNA expression of LASS2 in four bladder carcinoma cell lines using the methods of qPCR and western blotting.

We also demonstrated that LASS2 expression correlated with the biological characteristics of these human bladder carcinoma cell lines. The results suggest that LASS2 expression is downregulated in cell lines with a high degree of malignancy and that the more malignant tumor cells expressed lower amounts of LASS2 at the protein and mRNA levels. This study supports the role of LASS2 as a metastasis suppressor gene and its potential utility as a clinical prognostic marker in human bladder carcinoma.
The results of qPCR are coincidental to the results of western blotting. LASS2 may be a useful indicator of tumor invasion and progression. However, due to the increasing appreciation of the importance of LASS2 in human bladder carcinoma, as well as other cancers, future studies focusing on how LASS2 is regulated and the mechanisms of its anti-metastatic functions, with the goal of critically examining the possibilities of exploiting LASS2 as a therapeutic target, are required.

In conclusion, using qPCR and western blotting, we have shown that LASS2 expression may be correlated with the development and progression of human bladder cancer and may be a prognostic indicator for this cancer.

\section{Acknowledgements}

This study was supported by the National Natural Science Foundation of China (No. 81260374) and Doctor Innovation Fund of Kunming Medical University (No. 2012D03). The authors would like to thank Professor Hongyi $\mathrm{Xu}$ and Professor Yongtang Zheng for their assistance in preparing this manuscript.

\section{References}

1. Morgan TM, Keegan KA and Clark PE: Bladder cancer. Curr Opin Oncol 23: 275-282, 2011.

2. Valastyan S and Weinberg RA: Tumor metastasis: molecular insights and evolving paradigms. Cell 147: 275-292, 2011.

3. Saffar H, Sanii S, Heshmat R, et al: Expression of galectin-3, $\mathrm{nm}-23$, and cyclooxygenase- 2 could potentially discriminate between benign and malignant pheochromocytoma. Am J Clin Pathol 135: 454-460, 2011.

4. Kim SH, Lee SY, Park HR, et al: Nuclear localization of Nm23-H1 in head and neck squamous cell carcinoma is associated with radiation resistance. Cancer 117: 1864-1873, 2011.

5. Polanski R, Maguire M, Nield PC, et al: MDM2 interacts with NME2 (non-metastatic cells 2, protein), and suppresses the ability of NME2 to negatively regulate cell motility. Carcinogenesis 32: 1133-1142, 2011.

6. Jiang WX, Song BG and Wang PJ: Expression of nm23, KAI1 and spiral computed tomography findings in primary gallbladder carcinoma. Chin Med J (Engl) 122: 2666-2668, 2009.

7. Boissan M and Lacombe ML: NM23, an example of a metastasis suppressor gene. Bull Cancer 99: 431-440, 2012 (In French).

8. Cao GL, Chu MX, Fang L, et al: Analysis on DNA sequence of KiSS-1 gene and its association with litter size in goats. Mol Biol Rep 37: 3921-3929, 2010.

9. Hiney JK, Srivastava VK and Les Dees W: Insulin-like growth factor-1 stimulation of hypothalamic KiSS-1 gene expression is mediated by Akt: effect of alcohol. Neuroscience 166: 625-632, 2010.

10. Lee KH and Kim JR: Kiss-1 suppresses MMP-9 expression by activating p38 MAP kinase in human stomach cancer. Oncol Res 18: 107-116, 2009.

11. Shoji I, Hirose T, Mori N, et al: Expression of kisspeptins and kisspeptin receptor in the kidney of chronic renal failure rats. Peptides 31: 1920-1925, 2010.

12. Moon HG, Jeong SH, Ju YT, et al: Up-regulation of RhoGDI2 in human breast cancer and its prognostic implications. Cancer Res Treat 42: 151-156, 2010.

13. Zheng Z,Li J,HeX, et al: Involvement of RhoGDI2 in the resistance of colon cancer cells to 5-fluorouracil. Hepatogastroenterology 57: 1106-1112, 2010.

14. Niu H, Li H, Xu C and He P: Expression profile of RhoGDI2 in lung cancers and role of RhoGDI2 in lung cancer metastasis. Oncol Rep 24: 465-471, 2010.

15. Su J, You JF, Wang JL, et al: Overexpression of tumor metastasis suppressor gene 1 suppresses proliferation and invasion, but enhances apoptosis of human breast cancer cells MDA-MB-231 cells. Zhonghua Bing Li Xue Za Zhi 36: 672-676, 2007 (In Chinese). 
16. Su J, You JF, Wang JL, et al: Overexpression of human tumor metastasis-related gene TMSG-1 suppresses cell proliferation and invasion of a highly metastatic prostate cancer cell line PC-3M-1E8 in vitro. Zhonghua Zhong Liu Za Zhi 30: 404-407, 2008 (In Chinese)

17. Chen SH, Bubb MR, Yarmola EG, et al: Vacuolar $\mathrm{H}^{+}$-ATPase binding to microfilaments: regulation in response to phosphatidylinositol 3-kinase activity and detailed characterization of the actin-binding site in subunit B. J Biol Chem 279: 7988-7998, 2004.

18. Schiffmann S, Sandner J, Birod K, et al: Ceramide synthases and ceramide levels are increased in breast cancer tissue. Carcinogenesis 30: 745-752, 2009.

19. Wang H, Wang J, Zuo Y, et al: Expression and prognostic significance of a new tumor metastasis suppressor gene LASS2 in human bladder carcinoma. Med Oncol 29: 1921-1927, 2012.

20. Wang H, Yang D, Wang K and Wang J: Expression and potential role of chemokine receptor CXCR4 in human bladder carcinoma cell lines with different metastatic ability. Mol Med Rep 4: 525-528, 2011.

21. Yang D, Wang H, Wang J, et al: Establishment of a fluorescent implantation metastasis model of bladder cancer and real-time microscopic detection in nude mice. Asian Pac J Cancer Prev 12: 393-396, 2011.
22. Girnita A, All-Ericsson C, Economou MA, et al: The insulin-like grow th factor-I receptor inhibitor picropodophyllin causes tumor regression and attenuates mechanisms involved in invasion of uveal melanoma cells. Clin Cancer Res 12: 1383-1391, 2006.

23. Masek T, Vopalensky V, Suchomelova P and Pospisek M: Denaturing RNA electrophoresis in TAE agarose gels. Anal Biochem 336: 46-50, 2005.

24. Livak KJ and Schmittgen TD: Analysis of relative gene expression data using real-time quantitative PCR and the 2(-Delta Delta C(T)) method. Methods 25: 402-408, 2001.

25. Baron V, Adamson ED, Calogero A, et al: The transcription factor Egr1 is a direct regulator of multiple tumor suppressors including TGFbeta1, PTEN, p53, and fibronectin. Cancer Gene Ther 13: 115-124, 2006.

26. Calogero A, Arcella A, De Gregorio G, et al: The early growth response gene EGR-1 behaves as a suppressor gene that is downregulated independent of $\mathrm{ARF} / \mathrm{Mdm} 2$ but not $\mathrm{p} 53$ alterations in fresh human gliomas. Clin Cancer Res 7: 2788-2796, 2001.

27. Liotta LA, Steeg PS and Stetler-Stevenson WG: Cancer metastasis and angiogenesis: an imbalance of positive and negative regulation. Cell 64: 327-336, 1991.

28. Ichikawa T, Ichikawa Y and Isaacs JT: Genetic factors and metastatic potential of prostatic cancer. Cancer Surv 11: 35-42, 1991. 